\section{Determinantes próximos de la fecundidad: comportamiento reproductivo de las indígenas Chamibida de Antioquia, Colombia}

\author{
Proximal determinants of fertility: \\ reproductive behavior among Chamibida \\ indigenous women in Antioquia, Colombia
}

\author{
${ }^{1}$ Facultad de Enfermería, \\ Universidad de Antioquia, \\ Medellin, Colombia \\ Correspondencia \\ M. M. Arias-Valencia \\ Facultad de Enfermería, \\ Universidad de Antioquia. \\ Carrera 43 \# 23-53, apto 202, \\ Medellín, Colombia. \\ mariamav@tone.udea.edu.co \\ dgiraldoa@epm.net.co
}

\begin{abstract}
The aim of this study was to determine the reproductive timing used by Chamibida indigenous women in Antioquia, Colombia. This socio-demographic study used the reproductive history technique and was conducted in the year 2000. Two hundred and thirty-four homes on the Cristiania indigenous reservation were visited to obtain 253 reproductive histories (83.1\% of the women ages 15 to 49). Reproductive behavior was characterized by sexual debut at age 17.8 years (mean), with short intergenesic intervals in $69.0 \%$ and an adjusted fertility rate of 5.12 children. This reproductive pattern differs from all other indigenous groups and subgroups in Antioquia. As an explanation, Chamibida women are familiar with and accept Western birth control methods, besides having more access to formal schooling. Current Colombian health system policies are far from appropriate, since they fail to take the indigenous groups' specificities into account.
\end{abstract}

Demography; Fertility; South American Indians; Ethnic Groups
Maria Mercedes Arias-Valencia 1

\section{Introducción}

Los perfiles de fecundidad de los pueblos indígenas son poco conocidos, como señalan Coimbra Jr. \& Santos 1, por la ausencia de censos, de otras encuestas, registros regulares y por la precariedad de las fuentes 2 y sistemas de información que registren la especificidad por etnia.

En Colombia se destacan los estudios sobre demografía y salud de pueblos indígenas de Alcaraz et al. 3 y Arenas et al. 4; sobre fecundidad, Gálvez et al. 5, Acevedo 6, Galeano \& Alcaraz 7, Marín 8, así como F. Urrea (Reflexiones a Partir de los Estudios sobre Culturas Médicas Populares en Colombia. Su Lugar en la Investigación Sociomédica. Ponencia presentada en el Séptimo Congreso Colombiano de Antropología; 2004). En 1993 se incluyó, por primera vez, el componente étnico en el censo ${ }^{9}$. Sobre dicha base, el Instituto Nacional de Salud llevó a cabo la encuesta: Conocimientos, Actitudes y Prácticas en Salud en Comunidades Indígenas - CAPS, que incluye un componente demográfico 10.

Los trabajos referidos sobre demografía de microsociedades se suman a los realizados por Early \& Peters 11, Flowers 12, Santos et al. 13, Souza \& Santos 14 y Pagliaro 2; en Centroamérica, Daltabuit et al. 15; en las Américas, Coloma 16 levantó, en 1993, los diferenciales de morbimortalidad para los pueblos indígenas.

La demografía posee herramientas para analizar fenómenos sociales situándose, desde una 
perspectiva más amplia, en las condiciones en que se desenvuelve la reproducción. Este trabajo discute sobre demografía y salud reproductiva, y, en el área de la demografía antropológica, levanta el comportamiento reproductivo de una microsociedad. Con ello se pretende aportar más exploración a un área que la necesita, como lo expresa Berquó 17 (p. 81) cuando señala que "conceptos como, por ejemplo, reproducción como un constructo social y género como una relación política y de poder, centrales en la comprensión de la fecundidad y en la arena de las políticas de población, no han formado parte de la demografía".

El objetivo es caracterizar la fecundidad en las mujeres Embera Chamibida de Cristianía, compararlo con otros estudios que realicé con los Chamibida, que permiten documentar las modificaciones en los patrones reproductivos a lo largo del tiempo y compararlo con las demás etnias y subgrupos de Antioquia.

Este texto tiene relevancia para la salud colectiva, pues pretende aportar en la comprensión cuestiones relacionadas con la vida y los derechos reproductivos de los pueblos indígenas y constituye un insumo para la formulación de políticas, programas y servicios de salud con pertinencia social y adecuación cultural.

\section{Métodos y procedimientos}

A continuación, describiré el área y la población de estudio y los procedimientos de recolección y procesamiento de la información demográfica.

\section{Área y población de estudio}

Los indígenas Chamibida estudiados viven en Cristianía, en la región de Antioquia, localizada en la parte noroccidental de Colombia, con una extensión de $63.612 \mathrm{~km}^{2}$ de superficie (Figura 1). La topografía regional es bastante quebrada, atravesada por las cordilleras Central y Occidental de los Andes. Se caracteriza por la inseguridad persistente agudizada desde la década de los 70 hasta hoy ${ }^{18}$ y, también, por la exclusión basada en una representación de cultura hegemónica descendiente de los españoles. Fernández et al. 19 (p. 28) relatan que la población de Cristianía quedó "rodeada por los colonos de origen europoide".

Los indígenas de Antioquia forman parte de los llamados indígenas de tierras bajas, diferentes etnográficamente de los indígenas andinos. En total habitan 14.903 indígenas 20 pertenecientes a tres etnias: los Embera, los Tule (kuna) y los Zenú; los Embera comprenden tres subgrupos, los Eyabida, habitantes de la cordillera, los Dobida, de las riberas de los ríos y los Chamibida, de la cordillera en el suroeste antioqueño. Estudios anteriores muestran los indígenas de Antioquia con tasas de fecundidad elevadas, edades tempranas de inicio de la labor reproductiva y con intervalos intergenésicos (parto-inicio de gestación) cortos 5,21.

El trabajo se llevó a cabo en la reserva Chamibida de Cristianía, entre los municipios de Andes y Jardín, localizados en el suroeste antioqueño, con 39.404 y 15.649 habitantes respectivamente, a 1.350 y $1.750 \mathrm{mts}$. sobre el nivel del mar y a 121 y $138 \mathrm{~km}$ desde la capital 22 . Según el Artículo 21 del Decreto 2.164 de 1995, "la reserva es una institución legal y sociopolítica de carácter especial, conformada por una o más comunidades indígenas, que con un título de propiedad colectiva goza de las garantías de la propiedad privada, poseen su territorio y se rigen para el manejo de ésta y su vida interna por una organización autónoma amparada por el fuero indígena y su sistema normativo propio" 20.

Los indígenas, de la etnia embera, subgrupo Chamibida, son en total 1.374, conforman 234 familias, ocupan 407 hectáreas y representan $8,7 \%$ de la población del municipio. Los indígenas de esta reserva son representativos del subgrupo Chamibida con una proporción de $64,4 \%$ respecto de los 2.139 Chamibida de Antioquia.

La economía, igual que en otros asentamientos indígenas del suroeste antioqueño, se sustenta en las actividades productivas del cultivo cafetero. Según Marín 8, la estacionalidad del café y la dependencia económica del monocultivo le imprimen un carácter de inestabilidad y vulnerabilidad.

La comunidad está organizada alrededor de una Junta Administradora, un Consejo de Conciliación y Justicia y varios comités. A dicha organización, se superponen las parentelas tradicionales formando una parentela mayor, como dice uno de sus líderes: aquí todos somos familia; según Tönnies 23 , este tipo de comunidad cumple con las características de las comunidades de sangre.

Los indígenas tienen acceso al hospital de Jardín, con servicio de urgencias, hospitalización con 20 camas y consulta externa. La carretera asfaltada que une los dos municipios atraviesa la reserva. El tiempo de transporte entre la reserva y el hospital es de 20 minutos. Allí acuden para consulta por morbilidad, atención hospitalaria del parto y programas materno-infantiles, como inmunizaciones y planificación familiar. 


\section{Figura 1}

Localización de la Reserva Indígena de Cristianía, Municipio de Jardín, Antioquia, Colombia.

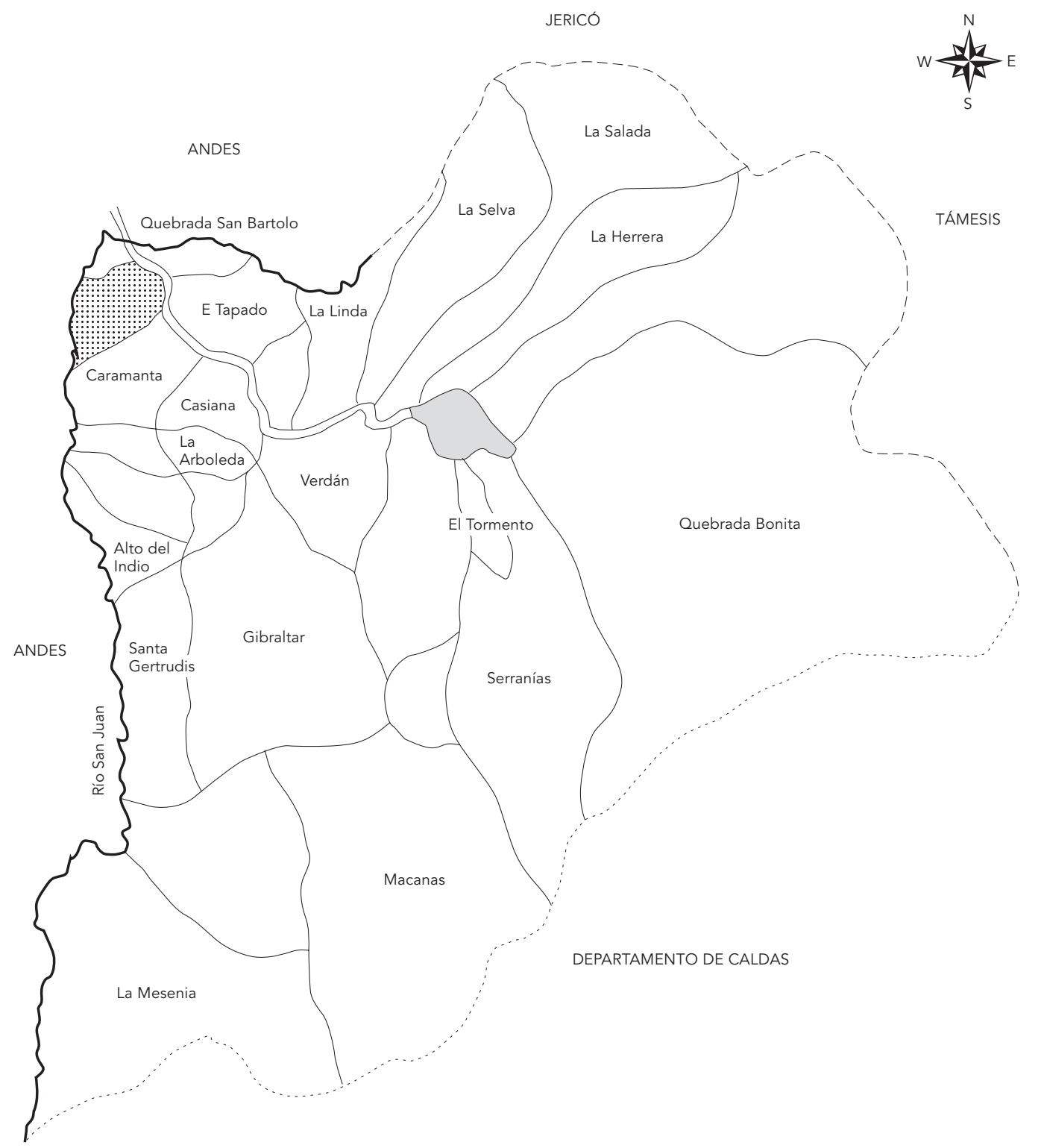
Convenciones
- - Límite Departamental
-. Límite Municipal
— Límite Veredal
= Carretera Troncal del Café
Cabecera Municipal
Resguardo de Cristianía

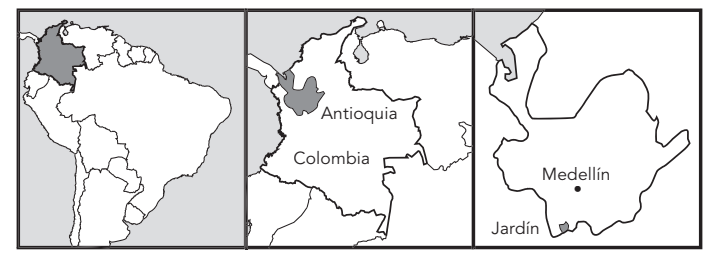


En referencia al saneamiento, la reserva no posee alcantarillado, existe un acueducto para 16 de las 234 viviendas. En cuanto a la disposición intradomiciliar de excretas, $39,0 \%$ poseen taza sanitaria, 37,9\% sanitario, 3,33\% pozo séptico y $2,1 \%$ letrina. Las basuras se tiran en campo abierto en $84,0 \%$ de las viviendas, se entierran en $13,33 \%$ y se arrojan a un pozo en $0,4 \%$.

Durante el trabajo identificamos tres tipos de condiciones externas: (a) la inseguridad, (b) la marginalidad y la pobreza, y, (c) la exclusión. (a) La inseguridad se debe al conflicto armado que sufre Colombia y también a la erosión de la tierra y las fallas geológicas que atraviesan la reserva y los municipios vecinos, en los cuales ya han ocasionado tragedias. En Cristianía ha habido deslizamientos de tierra, pérdida de cultivos y de infraestructura, desviación de los lechos de agua, agrietamiento y ondulaciones en el terreno.

(b) La marginalidad y la pobreza, relacionadas mutuamente, son consecuencia de los procesos de colonización que llevaron a que la reserva de Cristianía sea la tercera más pequeña de todo el territorio nacional 22 . Los domicilios están agrupados, con espacios peridomiciliarios pequeños, contrariando el patrón de asentamiento embera disperso, etnia de la que los Chamibida son un subgrupo. Los recursos bióticos son escasos, ha disminuido la producción de productos de pan-coger, tales como plátano - símbolo embera, fríjol, maíz y yuca y hay deterioro de las fuentes de agua debido a la sobreexplotación de la tierra, como ilustra Marín 8 (p. 26): "la caficultura desencadenó una utilización irracional de los recursos naturales con sobreexplotación de la tierra, llegando a la tala y quema del poco bosque que queda con el fin de ampliar las áreas de los cafetales que, se encuentran hasta en las pendientes montañosas de 45 grados de inclinación existentes en la reserva".

(c) La exclusión, tercera condición del contexto externo, se expresa como negación de la igualdad de derechos de atención por el Estado y de obtención de servicios y recursos.

\section{Recolección y procesamiento} de la información

Para demografía y salud, la vida reproductiva o período reproductivo potencial, comienza con la menarca y finaliza con la menopausia. En el presente trabajo, la vida reproductiva se tomó como período reproductivo con inicio, desarrollo y fin.

El instrumento demográfico que se aplicó fue la historia reproductiva, partiendo de la historia de nacimientos. Dicho instrumento fue probado, validado y ajustado. En el año 2000, se visitaron todas las 234 viviendas de Cristianía para obtener 253 historias reproductivas, correspondientes a $83,1 \%$ de las mujeres de 15 a 49 años. A cada mujer se le preguntó cuántos hijos e hijas nacidos vivos viven con ella, cuántos viven en otro lugar y cuántos murieron; cuántos abortos y mortinatos ha tenido durante su vida reproductiva. Se investigó la historia de cada hijo nacido vivo y de cada uno de los eventos obstétricos desde la menarca hasta el día de la encuesta.

Las fuentes de información sobre abortos fueron las historias reproductivas de las mujeres y los registros hospitalarios correspondientes a los años 1999 y 2000. En este subgrupo étnico no hubo restricciones abiertas para hablar de abortos y muertes de hijos, a pesar de las advertencias de la religión católica sobre la pecaminosidad de los abortos. Aún así, no se descarta la subrepresentación de las muertes fetales y neonatales. A partir de las historias reproductivas nos acercamos a los determinantes próximos e hicimos las estimaciones de fecundidad. En el análisis se cuidaron los criterios de comparabilidad con estudios anteriores que utilizan las historias reproductivas y asumen las mismas definiciones demográficas.

A pesar del alto grado de bilingüismo de los Chamibida de Cristianía y de la experiencia de campo, no se descartan errores resultantes de la formulación o del entendimiento de las preguntas, errores de codificación y de procesamiento. Éste incluyó procedimientos manuales, como revisión y verificación de la información, análisis de inconsistencias y detección de errores; procedimientos automáticos para la elaboración y depuración de las bases de datos con ayuda del programa Excel y el análisis estadístico de los resultados con ayuda del programa SPSS.

En todo momento guardé la confidencialidad, custodiando la información y manteniéndola en lugar seguro, cuidando el anonimato de las personas y omitiendo cualquier dato que pudiera identificar a los participantes. No hubo necesidad de trabajar con traductores debido al alto bilingüismo. Previa y posteriormente a la salida del campo, di informes e hice presentaciones de los hallazgos en la sede de la organización regional y en la reserva local.

\section{Resultados demográficos: la fecundidad}

A continuación se presentan las tasas de fecundidad (quantum) y el calendario reproductivo (timing): menarca, nupcialidad, entrada a la 
unión, gravidez, parto, intervalos intergenésicos, lactancia y menopausia.

En el año 2000, la tasa bruta de natalidad (TBN) fue 34,21 por 1.000 habitantes. La tasa general de fecundidad (TGF) fue 140,7 nacidos vivos por 1.000 mujeres en edad fértil (MEF). La tasa total de fecundidad (TFT), fue 5,79 hijos en el 2000 y la tasa ajustada 5,12 hijos (Tabla 1).

El promedio de edad de la menarca fue 13,5 años, con edades mínima y máxima de 9 y 19 años (Tabla 2). Las mujeres alguna vez unidas, fueron $85,3 \%$, las solteras, $14,7 \%$. Dentro de las mujeres con hijos se incluyen 7 separadas $(2,0 \%)$. La mayoría de las mujeres $(71,7 \%)$ había tenido una unión conyugal durante su vida, 34 habían experimentado dos (9,6\%), y 2,0\%, 3 uniones. Los estatus de padre y madre son mayoritarios.

La edad de entrada a la unión (Tabla 3) presenta las siguientes proporciones: de 10 a 14 años $16,4 \%$, de 15 a 19 años 59,1\%, de 20 a 24 años 19,4\%, y de 25 y más años 5,0\%. Entre los 14 y los 18 años, se presenta la entrada masiva a la unión, la moda se sitúa en los 15 años, la mediana en los 17 años, y, el promedio fue 17,8 años, con valores mínimo y máximo de 11 y 33 . En la Tabla 4 se observa, en un período de cinco años (1995 a 2000), desplazamiento de un año en la edad mínima de entrada a la unión, disminución en la proporción de mujeres que comenzaron la unión conyugal a los 12 años o menos y desplazamiento de 7 años en la edad máxima.

La proporción de mujeres que iniciaron la primera gestación hasta los 18 años fue $38,5 \%$ (Tabla 3). Las mujeres sin experimentar gravidez fueron 3,7\% (Tabla 5); 17,3\% de las mujeres habían experimentado una gestación y $13,3 \%$ dos; $65,7 \%$ fue la proporción de multigrávidas con 3 a 16 gestaciones, que fue el valor máximo.

La edad más temprana del primer parto fue de 12,7 años (Tabla 2). La edad promedio fue 19,7 años; la mitad de las mujeres, 51,6\% de ellas (Tabla 3) fueron madres antes de los 20 años y 4,8\% antes de los 15 años; 20,7\% de ellas habían tenido su primer hijo a los 30 años o más. El promedio de partos domiciliarios fue 3,6, más de dos veces el promedio de partos hospitalarios, 1,4. El parto hospitalario ha desplazado progresivamente el parto domiciliario, lo cual tiene relación, entre otros factores, con la cercanía de la reserva de los términos municipales.

Pese a la posible subrepresentación de los abortos y los mortinatos, la proporción de mujeres que durante su vida reproductiva tuvo abortos fue $21,8 \%$, y la proporción de ellas que relató haber tenido mortinatos $6,1 \%$. La razón de abortos, 250 por 1.000 nacidos vivos en 1999 - año en que hubo 10 abortos -, y 85,1 por 1.000 nacidos vivos en el 2000.
Tabla 1

Medidas sintéticas de fecundidad de mujeres Embera Chamibida.

Reserva Indígena de Cristianía, Antioquia, Colombia, 2000.

\begin{tabular}{lr}
\hline Indicador & \\
\hline Tasa bruta de natalidad (x 1.000 habitantes) & 34,21 \\
Tasa general de fecundidad (x 1.000 mujeres en edad fértil) & 140,70 \\
Tasa global de fecundidad (hijos) & 5,79 \\
Tasa global de fecundidad ajustada (hijos) & 5,12 \\
\hline
\end{tabular}

Hasta el momento de la encuesta, 69,0\% había tenido en promedio, durante su vida reproductiva, intervalos intergenésicos menores de 24 meses (Figura 2). Ellos tienen relación con el tiempo de lactancia y con los métodos de espaciamiento de embarazos. Aún en Cristianía, cercana a dos términos municipales, el promedio de lactancia es de 16,69 meses. La mayoría de las mujeres amamantan entre 6 meses y dos años. Es preocupante la disminución en el tiempo de lactancia, a juzgar por $10,9 \%$ de las mujeres que lactaron menos de 6 meses y 17,75\% de 6 a 12 meses. Hasta la fecha de la encuesta 49,5\% habían utilizado algún método de planificación familiar durante su vida reproductiva. Los anovulatorios orales más frecuentemente, $14,2 \%$, y la tubectomía $11,9 \%$.

Respecto de la última edad reproductiva, observamos una separación de 8,3 años entre los promedios de edad del último evento reproductivo, 37,9 años, y de edad de ausencia de menstruación, en 46,2 años, relacionada con la esterilización voluntaria.

\section{Discusión}

La TGF en los Chamibida (140,7 x 1.000 MEF) es menor que la de otros pueblos indígenas, a saber, 224, 1 x 1.000 MEF que reportamos para los Eyabida en 1985 3; 209,9 x 1.000 MEF (ajustada) para las etnias de Antioquia en 1995 5; en pueblos indígenas de Brasil, Alcaraz 24 reporta valores superiores a 200 en las últimas 4 décadas. Si bien la TGF en los Chamibida es expresivamente lejana de las etnias de Antioquia, su valor es superior a 100, considerado alto por la Organización Mundial de la Salud (OMS). En Colombia se reportó una tasa de 91 nacidos vivos x $1.000 \mathrm{MEF} 25$. Igual ocurre con las demás medidas de fecundidad, a saber, las tasas de natalidad y de fecundidad total.

Las tasas específicas de fecundidad encontradas en el año 2000 son consistentes con las 
Tabla 2

Calendario reproductivo (timing) de mujeres Embera Chamibida. Reserva Indígena de Cristianía, Antioquia, Colombia, 2000.

\begin{tabular}{lccccc}
\hline & N válido & Mínimo & Máximo & Media & Desv. Típ. \\
\hline Edad de la menarca & 280 & 9,0 & 19,0 & 13,5 & 1,7 \\
Entrada a la unión & 299 & 11,0 & 33,0 & 17,8 & 3,8 \\
Edad de la primera gestación & 290 & 11,9 & 38,7 & 3,9 & 4,1 \\
Edad en el primer parto & 289 & 12,7 & 39,4 & 4,1 \\
\hline
\end{tabular}

Tabla 3

Calendario reproductivo (timing) de mujeres Embera Chamibida. Reserva Indígena de Cristianía, Antioquia, Colombia, 2000.

\begin{tabular}{|c|c|c|c|c|c|c|c|c|c|}
\hline \multirow[t]{2}{*}{ Edad } & \multicolumn{3}{|c|}{ Entrada a la unión } & \multicolumn{3}{|c|}{ Inicio de la 1ạ gestación } & \multicolumn{3}{|c|}{ Edad del primer parto } \\
\hline & Frecuencia & $\%$ & $\%$ acumulado & Frecuencia & $\%$ & $\%$ acumulado & Frecuencia & $\%$ & $\%$ acumulado \\
\hline 11 & 2 & 0,7 & 0,7 & 1 & 0,3 & 0,3 & & & \\
\hline 12 & 6 & 2,0 & 2,7 & 1 & 0,3 & 0,6 & 2 & 0,6 & 0,6 \\
\hline 13 & 10 & 3,4 & 6,0 & 1 & 0,3 & 0,8 & 3 & 0,8 & 1,4 \\
\hline 14 & 31 & 10,4 & 16,4 & 9 & 2,5 & 3,4 & 12 & 3,4 & 4,8 \\
\hline 15 & 39 & 13,1 & 29,5 & 24 & 6,8 & 10,2 & 1 & 0,3 & 5,1 \\
\hline 16 & 38 & 12,8 & 42,3 & 31 & 8,8 & 19,0 & 24 & 6,8 & 11,9 \\
\hline 17 & 38 & 12,8 & 55,0 & 35 & 9,9 & 28,9 & 37 & 10,5 & 22,4 \\
\hline 18 & 32 & 10,7 & 65,8 & 34 & 9,6 & 38,5 & 32 & 9,1 & 31,4 \\
\hline 19 & 29 & 9,7 & 75,5 & 1 & 0,3 & 38,8 & 30 & 8,5 & 39,9 \\
\hline 20 & 19 & 6,4 & 81,9 & 32 & 9,1 & 47,9 & 41 & 11,6 & 51,6 \\
\hline 21 & 16 & 5,4 & 87,2 & 30 & 8,5 & 56,4 & 22 & 6,2 & 57,8 \\
\hline 22 & 7 & 2,3 & 89,6 & 25 & 7,1 & 63,5 & 26 & 7,4 & 65,2 \\
\hline 23 & 7 & 2,3 & 91,9 & 18 & 5,1 & 68,6 & 1 & 0,3 & 65,4 \\
\hline 24 & 9 & 3,0 & 95,0 & 6 & 1,7 & 70,3 & 11 & 3,1 & 68,6 \\
\hline 25 & 2 & 0,7 & 95,6 & 16 & 4,5 & 74,8 & 8 & 2,3 & 70,8 \\
\hline 26 & 1 & 0,3 & 96,0 & 4 & 1,1 & 75,9 & 14 & 4,0 & 74,8 \\
\hline 27 & 2 & 0,7 & 96,6 & 4 & 1,1 & 77,1 & 4 & 1,1 & 75,9 \\
\hline 29 & 3 & 1,0 & 97,7 & 6 & 1,7 & 78,8 & 1 & 0,3 & 76,2 \\
\hline 30 & 4 & 1,3 & 99,0 & 2 & 0,6 & 79,3 & 2 & 0,6 & 76,8 \\
\hline 31 & 1 & 0,3 & 99,3 & 3 & 0,8 & 80,2 & 6 & 1,7 & 78,5 \\
\hline 32 & 1 & 0,3 & 99,7 & 1 & 0,3 & 80,5 & 2 & 0,6 & 79,0 \\
\hline 33 & 1 & 0,3 & 100,0 & 3 & 0,8 & 81,3 & 1 & 0,3 & 79,3 \\
\hline \multirow[t]{3}{*}{ Total } & 298 & 100,0 & & 1 & 0,3 & 81,6 & 2 & 0,6 & 79,9 \\
\hline & & & & 1 & 0,3 & 81,9 & 2 & 0,6 & 80,5 \\
\hline & & & & 1 & 0,3 & 82,2 & 2 & 0,6 & 81,0 \\
\hline Sin gestación & & & & 63 & 17,8 & 100,0 & 1 & 0,3 & 81,3 \\
\hline \multirow[t]{2}{*}{ Total } & & & & 353 & 100,0 & & 1 & 0,3 & 81,6 \\
\hline & & & & & & & 1 & 0,3 & 81,6 \\
\hline Sin parto & & & & & & & 64 & 18,1 & 100,0 \\
\hline Total & & & & & & & 353 & 100,0 & \\
\hline
\end{tabular}


encontradas en 1995-1996, se acercan más a las de la población rural colombiana y discrepan de las encontradas en las etnias de Antioquia. Esto significa que los cambios en el patrón de fecundidad Chamibida permanecen consistentemente en dos períodos de tiempo observados. En Colombia son prácticamente inexistentes los trabajos sobre calendario reproductivo (timing), en indígenas y no indígenas, con los cuales fuera posible hacer comparaciones.

Los estudiosos de la fecundidad se han pronunciado ante el significado de sus componentes: para Preston 26, estudioso de la relación mortalidad-fecundidad, es esencial considerar los determinantes próximos, es decir, los niveles, diferenciales y determinantes que inciden la fecundidad, y que actúan entre los factores macroeconómicos y la fecundidad 27. Bongaarts 28 identificó cuatro determinantes próximos, a saber: (1) la proporción de mujeres casadas, (2) el uso de anticonceptivos, (3) el aborto inducido y (4) la lactancia materna, que con el tiempo de posparto, constituye un período de infecundabilidad.

La proporción de mujeres solteras en Cristianía muestran cambios en el patrón de nupcialidad. En 1995-1996 se constató la ausencia de solteras mayores de 20 años en las Zenú, después de los 23 años en las Eyabida y Dobida y mayores de 26 años en las Chamibida y, con Alcaraz et al. 3, demostramos que el estatus de la soltería y la separación eran inexistentes en las Eyabida en 1985.

La primera unión se tomó como el inicio de las relaciones sexuales. Si se compara con etnias y subgrupos de Antioquia, se observa que, en 1995-1996, la edad de la entrada fue 12 años o menos en $24,0 \%$ de las Eyabida y Dobida, $12,7 \%$ de las Chamibida y 11,8\% de las Zenú. Una Chamibida tuvo su primera unión a los 10 años. Tuvieron su primera unión a los 16 años, 77,8\% de las Eyabida y Dobida, 63,0\% de las Chamibida y $84,2 \%$ de las Zenúes. La edad máxima fue 20 años en las Zenúes, 23 años en las Eyabida y Dobida y 26 años en las Chamibida.

El hecho de permanecer la sexualidad unida con la reproducción, para hombres y mujeres indígenas, marca diferencia con muchas sociedades y con la nuestra, en la cual, la edad de la primera unión se ha venido separando del comienzo de las relaciones sexuales 25 .

Si se tiene en cuenta el criterio, según el cual, el período de la adolescencia abarca hasta $\operatorname{los} 18$ años $29,31,4 \%$ fueron madres adolescentes, lo cual constituye marcada diferencia, respecto de las demás etnias y subgrupos de Antioquia que, en los años 1995-1996, presen-
Tabla 4

Comparación de las edades de entrada a la unión en mujeres Embera

Chamibida de la Reserva Indígena de Cristianía, año 2000, con la totalidad de las Chamibida de Antioquia, Colombia, 1995-1996.

\begin{tabular}{lcc}
\hline & $\begin{array}{c}\text { Chamibida } \\
\text { de Antioquia }\end{array}$ & $\begin{array}{c}\text { Chamibida } \\
\text { de Cristianía }\end{array}$ \\
\hline Edad mínima & 10 años & 11 años \\
12 años o menos & $12,7 \%$ & $2,8 \%$ \\
Edad máxima & 26 años & 33 años \\
\hline
\end{tabular}

Tabla 5

Gravidez de mujeres Embera Chamibida de la Reserva Indígena de Cristianía. Antioquia, Colombia, 2000.

\begin{tabular}{lrrr}
\hline & Frecuencia & \% válido & $\%$ acumulado \\
\hline 0 & 11 & 3,7 & 3,7 \\
1 & 52 & 17,3 & 20,9 \\
2 & 40 & 13,3 & 34,2 \\
3 & 37 & 12,3 & 46,5 \\
4 & 31 & 10,3 & 56,8 \\
5 & 34 & 11,3 & 68,1 \\
6 & 19 & 6,3 & 74,4 \\
7 & 10 & 3,3 & 77,7 \\
8 & 20 & 6,6 & 84,4 \\
9 & 9 & 3,0 & 87,4 \\
10 & 10 & 3,3 & 90,7 \\
11 & 13 & 4,3 & 95,0 \\
12 & 6 & 2,0 & 97,0 \\
13 & 1 & 0,3 & 97,3 \\
14 & 5 & 1,7 & 99,0 \\
15 & 1 & 0,3 & 99,3 \\
16 & 2 & 0,7 & 100,0 \\
Total & 100,0 & \\
\hline & & &
\end{tabular}

taban las siguientes proporciones: $72,7 \%$ de las embera Eyabida y Dobida, 46,3\% de las Chamibida y $80,3 \%$ de las Zenúes.

Ampliando las comparaciones, observamos que la proporción de mujeres Chamibida que inició su primera gestación antes de 15 años, fue $10,2 \%$, frente a $49,1 \%$ de las Eyabida y Dobida en 1995-1996, 23,6\% de las Chamibida y $31,4 \%$ de las Zenúes. En 1995, iniciaron antes de 20 años 94,5\% de las Eyabida y Dobida, $78,2 \%$ de las Chamibida y $90,1 \%$ de las Zenúes 30 , frente a $56,4 \%$ de las Chamibida de Cristinía en el año 2000. Lo anterior, refleja el desplazamiento de la edad de entrada a la unión. 
Figura 2

Distribución porcentual de los promedios de intervalos intergenésicos en mujeres

Embera Chamibida. Reserva Indígena de Cristianía, Antioquia, Colombia.

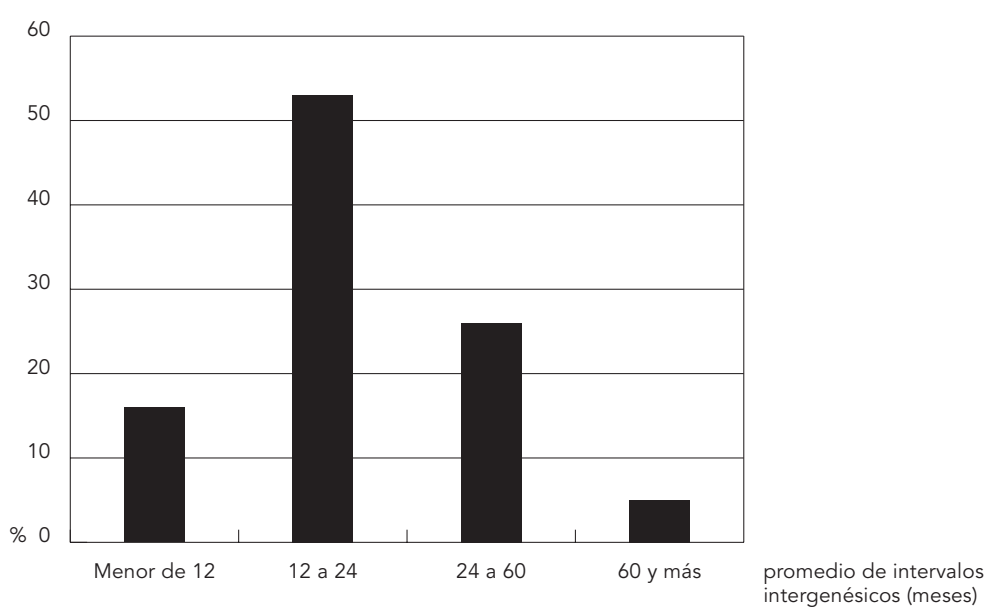

La razón de abortos es consistente con lo encontrado entre los Embera Eyabida de Dabeiba, Antioquia cuando, en 1985, registramos una razón de 92,59 abortos por 1.000 nacidos vivos ${ }^{3}$. En 1995-1996, más del 90,0\% de las mujeres, no relataron mortinatos. Este hecho contrasta con los datos de 1985 cuando reportamos, altos índices de mortalidad fetal ${ }^{3}$. Las dificultades para la obtención de información sobre abortos obedecen, como ya vimos, a las sanciones negativas, influidas principalmente por los discursos religiosos, y por las diferencias en la definición de estatus viviente del feto en las culturas indígenas 5. En Colombia se encuentran registros de muertes fetales hasta 1989. En 1990 se suspendió la recolección de dicha información 9 . Pagliaro 2 (p. 2), estudiando los pueblos indígenas de Brasil, considera que "ellos tienen dominio sobre el tamaño de la familia y de la población que desean para atender las necesidades inherentes al funcionamiento de sus complejos sistemas de organización social, a través de prácticas de restricción voluntaria de los nacimientos y prácticas abortivas naturales y mecánicas".

El calendario reproductivo (timing) se refleja en los intervalos intergenésicos (IIG) que revisten importancia, en la protección y recuperación materna después de los eventos obstétricos (partos o abortos), en la supervivencia infantil, en la lactancia y la crianza; son claves para la planificación familiar, que debería ade- cuarse a las especificidades étnicas encontradas en este y otros trabajos.

El cálculo de los IIG que consideró las muertes fetales, se hizo sobre el total de mujeres con más de un hijo. La alta proporciones de IIG cortos (69,0\%) es similar en los demás grupos, y superior en la totalidad del las Chamibida de Antioquia. En 1995-1996, 68,0\% de las mujeres Eyabida y Dobida, 48,7\% de las Chamibida y 73,9\% de las Zenú, había tenido en promedio durante su vida reproductiva, intervalos cortos - menores de 24 meses 30 . Lo mismo en 77,0\% de las Tule (Kuna).

La práctica lactancia materna ha sido una de las más resistentes y ha tenido gran importancia para la supervivencia indígena. Ella ha persistido a pesar de que el embate de las leches comerciales hizo impacto en la preparación en puericultura de médicos, otros profesionales y personal de salud. El tiempo promedio de lactancia fue 16,69 meses. Para las etnias de Antioquia, con Gálvez et al. 5, informamos 16 meses en 1995, con promedios de 15,6 en Embera Eyabida, 17,39 Embera Dobida, 12,62 Embera Chamibida, 13,3 Zenú y 21,0 meses en Tule (Kuna).

El aplazamiento de la edad al primer hijo, reflejo del desplazamiento de la entrada en la unión, y el uso de métodos anticoncepcionales han contribuido significativamente en el descenso de la fecundidad. La planificación familiar entre las Embera Chamibida marca una ruptura con la norma de los pueblos indígenas de reproducir el grupo 5. En Antioquia, los resultados de investigaciones anteriores, indican, de forma consistente, la baja limitación de gestaciones y la sanción negativa de la anticoncepción y el aborto. En contraste, las mujeres Chamibida conocen y aceptan la planificación familiar occidental y asisten al programa en la unidad hospitalaria 30 . Los métodos que refieren haber usado son: el ritmo, el retiro, los óvulos, los anovulatorios orales y parenterales que generalmente no se usan antes de la primera gestación, y el dispositivo intrauterino. Entre las molestias, ellas se quejan de cefalea asociada con los anovulatorios, hemorragias asociadas con el dispositivo intrauterino y mareos.

Así, las prácticas reproductivas, ejemplo de imperativos culturales en las sociedades indígenas e impermeables a la cultura occidental, fueron permeables a la planificación familiar, conservando el mandato masculino y contraviniendo las normas de la oficialidad católica que, en algunas posturas, propende por una fecundidad natural, siguiendo el imperativo de tener todos los hijos que Dios mande y, prohibiendo el control natal por medios no natura- 
les. La participación masculina es decisiva en la adhesión a la planificación familiar occidental: en las relaciones jerárquicas de género, el hombre es el que manda, pero los hombres chamibida mandaron a las mujeres planificarse.

El promedio de edad del primer parto continúa inferior a 20 años, similar a las etnias de Antioquia aunque con diferencias entre los grupos: en 1995-1996, la edad del primer parto en las Chamibida ocurrió en promedio, dos años más tarde que en las Eyabida y Dobida ( $\mathrm{p}=$ $0,0004)$ y 1,5 años más tarde que las Zenú ( $\mathrm{p}=$ $0,001) 31$.

Aunque las edades mínimas al primer parto son tempranas, la proporción de mujeres, cuyo primer parto fue antes de 15 años fue diferente respecto de los años 90: pasó de $11,0 \%$ en las Chamibida de Antioquia, para 4,8\% en las Chamibida de Cristianía en 2000. De las Eyabida y Dobida, 32,7\% lo tuvieron antes de 15 años, $11,0 \%$ de las Chamí y 36,6\% de las Zenú. Edades tempranas también encontró Daltabuit 15 en las Mayas de Yalcobá-Yucatán en México, con $58,0 \%$ entre los 16 y los 20 años.

El desplazamiento del parto domiciliario por el hospitalario, muestra el cambio cultural de la sociedad Chamibida frente a las etnias de Antioquia. Esta es una señal de la adhesión a los programas estatales, que, durante las últimas cuatro décadas, han pretendido aumentar la cobertura del parto institucional-hospitalario. Los costos culturales son difíciles de comprender en su verdadera dimensión, pero se pueden observar algunos de sus posibles efectos. El primero y más importante es la disminución de la autonomía, pues de la autosuficiencia para los eventos de la vida (nacimiento y muerte) se pasa a depender de una cultura ajena que desvaloriza el imaginario cultural indígena. En segundo lugar, se enajenan saberes especializados como la partería, que está desapareciendo en los Chamibida - en los Eyabida y Dobida no existe la partería como oficio. Cada mujer se forma como partera a través de los eventos de su cuerpo y cada madre va a ser partera de sus hijas, acompañada o no del marido y futuro padre.

Un parto hospitalario era impensable para los eyabida en la década de los 80 , pues representaría la máxima agresión al pudor femenino, debido a su connotación erótica. Finalmente, la dependencia del sistema hospitalario genera otras dependencias, tales como del sistema de transporte, que es oneroso en tiempo y dinero. Otros eventos del ciclo vital están enajenados culturalmente e institucionalizados como sacramentos de la religión católica, a saber, el nacimiento por el bautismo, la puber- tad por el matrimonio, y la muerte por el entierro católico, con altas erogaciones económicas.

¿Qué piensan las Chamibida del parto hospitalario? En la investigación de campo se pudo observar que las mujeres forman una decisión acerca del lugar del parto con ayuda de las parteras quienes se esfuerzan por detectar algún peligro y las aconsejan, basadas, tanto sus conocimientos como en la capacitación que recibieron de la institución hospitalaria. Sin embargo, uno de los hechos más recordados fue una muerte materna reciente, que fue aludida repetidamente, como motivo de búsqueda de atención hospitalaria del parto. Actualmente no se escuchan quejas de la forma de atención, y el parto hospitalario forma parte del cotidiano de los Chamibida. En esto han contribuido los promotores chamibida de salud, que, formados a la manera occidental, favorecen directa o indirectamente el parto hospitalario. Módena 31 (p. 156) ilustra acerca de cómo las instituciones intervienen en el desplazamiento y enajenación del imaginario cultural: “(...) el centro de salud contribuye, junto con otras instancias sociales (farmacias, médicos privados, medios de comunicación...), a penetrar en prácticas y concepciones, con técnicas y procedimientos del sistema médico científico. (...) Ha salvado niños y madres en partos difíciles y ha introducido procedimientos, no necesariamente los mejores, en las técnicas de las parteras empíricas".

No es poca la responsabilidad del sistema de salud en el desplazamiento del saber tradicional; además, existe una distancia real entre las pérdidas en la autonomía para enfrentar los eventos de la vida, y una atención adecuada a las especificidades étnicas que compense la enajenación cultural.

La escolaridad debe analizarse en relación con los cambios en la fecundidad 32. Actualmente, los Chamibida de Cristianía representan el único subgrupo étnico en Antioquia con una diferencia en el acceso y en el nivel de escolaridad formal (Tabla 6). Al comparar los datos con las mujeres embera de 15 a 24 años, en la década anterior, el acceso a la escolaridad formal aumentó significativamente durante la segunda mitad del siglo veinte $(\mathrm{p}=0,000)$.

\section{Consideraciones finales}

La fecundidad de las mujeres Chamibida discrepa de los demás grupos étnicos de Antioquia y de Latinoamérica, pero es alta respecto de los valores nacionales. Su comportamiento reproductivo - que involucra decisiones entre opciones modeladas por normas sociales y ex- 
Tabla 6

Comparación de la escolaridad de mujeres Embera Chamibida de la Reserva Indígena de Cristianía, año 2000, con la totalidad de las mujeres indígenas de Antioquia, Colombia, 1995-1996.

\begin{tabular}{ccc}
\hline & $\begin{array}{c}\text { Embera } \\
\text { de Antioquia }\end{array}$ & $\begin{array}{c}\text { Chamibida } \\
\text { de Cristianía }\end{array}$ \\
\hline Sin escolaridad & 84,9 & 35,5 \\
1 & 1,9 & 8,5 \\
2 & 0,0 & 20,0 \\
3 & 9,6 & 9,7 \\
4 & 1,9 & 6,1 \\
5 & 19 & 9,1 \\
Secundaria & & \\
6 & & 2,1 \\
7 & & 2,4 \\
8 & & 3,3 \\
Más de 8 & & 3,3 \\
\hline
\end{tabular}

pectativas individuales y de pareja - representa una transformación en los patrones culturales étnicos, en una esfera de la vida que tradicionalmente no ha sido permeable, en tanto la reproducción está influenciada por las expectativas y las normas explícitas o tácitas de reproducción y de continuidad étnica, con las cuales está relacionado directamente el prestigio femenino 5 .

Los principales cambios se observaron el patrón de nupcialidad, la edad del primer parto, la atención del parto, la adhesión a la planificación familiar y la edad del último evento reproductivo. Los hombres y las mujeres separadas, $2,0 \%$, en conjunto con las mujeres solteras, $14,7 \%$, representan una categoría nueva, a pesar de la poca valorización que las sociedades indígenas dan a los estados diferentes de la conyugalidad y la filiación. Se observó un año de desplazamiento de la edad mínima de entrada a la unión, disminución de la entrada a la unión en edades tempranas, y un desplazamiento de 7 años en la edad máxima. Se encontró una disminución de un 50,0\% en la proporción de mujeres que tuvieron el primer parto antes de 15 años: en los años 90, 11,0\% y en el año 2000, 4,8\%; además, $20,7 \%$ de ellas habían tenido su primer hijo a los 30 años o más. El promedio de partos hospitalarios fue 1,4, desplazando el parto domiciliario influenciado por la cercanía de las cabeceras municipales y por la adhesión a los programas estatales, idea impensable, para el subgrupo eyabida en los años 80 .

Entre los factores relacionados con los cambios culturales se destacan, la escasez de territorio, las diferencias en el acceso a la educación y, la adhesión al sistema de salud occidental. La cuestión territorial se resalta como factor explicativo, ya que, como consecuencia de los procesos de colonización, la reserva de Cristianía es la tercera más pequeña de toda la nación 23. Las diferencias en el acceso a la educación que reporta la literatura como una de las variables mas relacionadas con la fecundidad, y que presenta diferencias frente a los demás grupos étnicos de Antioquia.

Se destaca el papel de los varones, en dos sentidos; primero, la conciencia de los líderes acerca del esfuerzo económico que representa la manutención de los hijos y, segundo, la decisiva participación masculina en la adhesión a la planificación familiar occidental, conservando la jerarquía de género, lo cual refleja las complejas relaciones intergenéricas, intergeneracionales y de poder.

Por último, aportamos en la mediación entre entre los factores contextuales, económicos y sociales, y la reproducción y, entre dos sistemas de normas culturales. Dicha complejidad debe ser atendida por los servicios de atención en salud que, presentan alto grado de desconsideración por las especificidades étnicas. Por el contrario, deberían trabajar en la adecuación cultural y reorientar la atención en salud, puesto que, para cuestiones complejas se requieren acciones integrales. 


\section{Resumen}

El objetivo de este artículo es caracterizar el calendario reproductivo en las Chamibida en el Departamento de Antioquia, Colombia. Este es un estudio sociodemográfico realizado en el año 2000 con la técnica de historia reproductiva. Se visitaron todas las $234 \mathrm{vi}$ viendas de la Reserva Indígena de Cristianía para obtener 253 historias reproductivas correspondientes a 83,1\% de las mujeres de 15 a 49 años. El comportamiento reproductivo se caracteriza por una entrada a la unión a los 17,8 años en promedio; intervalos intergenésicos cortos en 69,0\%, con una tasa de fecundidad ajustada de 5,12 hijos. Este patrón reproductivo es diferente frente a las demás etnias y subgrupos de Antioquia. Como explicación se destaca que de las mujeres chamibida conocen y aceptan la planificación familiar occidental y, tienen mayor acceso a la escolaridad formal. Las políticas actuales están lejos de ser apropiadas, en tanto pasan por alto la especificidad de estos grupos étnicos.

Demografía; Fertilidad; Indios Sudamericanos; Grupos Étnicos

\section{Agradecimientos}

La autora agradece especialmente a la Dra. Cristina de A. Possas y al Dr. Ricardo Ventura Santos por sus valiosas contribuciones en el desarrollo de la tesis doctoral y en la elaboración del presente artículo.

\section{Referencias}

1. Coimbra Jr. CEA, Santos RV. Perfil epidemiológico da população indígena no Brasil: considerações gerais. Porto Velho: Centro de Estudos em Saúde do Índio de Rondônia/Rio de Janeiro: Escola Nacional de Saúde Pública, Fundação Oswaldo Cruz; 2001. (Documento de Trabalho 3).

2. Pagliaro H. A revolução demográfica dos povos indígenas: a experiencia dos Kaiabi do Parque Indígena do Xingu - Mato Grosso 1970-1999 [Tese de Doutorado]. São Paulo: Universidade de São Paulo; 2002.

3. Alcaraz G. A fecundidade entre os guarani: um legado de Kunhankarai [Tese de Doutorado]. Rio de Janeiro: Escola Nacional de Saúde Pública, Fundação Oswaldo Cruz; 2000.

4. Arenas M. Encuesta de morbilidad realizada entre la comunidad indígena cuna de Caimán Nuevo Turbo - Antioquia Julio de 1975. Rev Esc Nac Salud Pública 1976; 2:44-5.

5. Gálvez A, Alcaraz G, Arias MM, Galeano A, Gutiérrez SY, López AD, et al. Estatus femenino y patrón de fecundidad en las etnias indígenas de Antioquia: informe final. Medellín: Universidad de Antioquia/Instituto Colombiano para el Desarollo de la Ciencia y la Tecnología; 1998.

6. Acevedo C. Salud reproductiva de la etnia zenú del Urabá antioqueño. Medellín: Facultad de Enfermería, Universidad de Antioquia; 1999.

7. Galeano A, Alcaraz G. El estatus de la mujer indígena Kuna de Antioquia y su relación con el comportamiento reproductivo. Medellín: Universidad de Antioquia; 1995.

8. Marín J. Los chamí del suroeste antioqueño: la Sucia y la María en perspectiva de futuro. Medellín: Departamento de Antropología, Facultad de Ciencias Sociales y Humanas, Universidad de Antioquia; 1997.

9. PROFAMILIA. Encuesta: investigación nacional de demografía y salud. Programa de encuestas de demografia y salud. Bogotá: Macro International; 1995.

10. Piñeros M, Ruiz M. Aspectos demográficos en comunidades indígenas de Colombia. Salud Pública Méx 1998; 40:324-9.

11. Early J, Peters J. The population dynamics of the Mucajai Yanomami. San Diego: Academic Press; 1990.

12. Flowers N. Crise e recuperação demográfica: os Xavante de Pimentel Barbosa, Mato Grosso. In: Santos RV, Coimbra Jr. CEA, organizadores. Saúde e povos indígenas. Rio de Janeiro: Editora Fiocruz, 1994. p. 94-117.

13. Santos RV, Flowers N, Coimbra Jr. CEA, Gugelmin S. Human ecology and health in the context of change: the xavante indians of Mato Groso, Brasil. In: Foller ML, Hansson LO, editors. Human ecology and health: adaptation to a changing world. Göteborg: Göteborg University, 1996. p. 94-117.

14. Souza LG, Santos RV. Perfil demográfico da população indígena Xavánte de Sangradouro - Volta Grande, Mato Grosso (1993-1997), Brasil. Cad Saúde Pública 2001; 17:355-65.

15. Daltabuit M. Mujeres mayas. Trabajo, nutrición y fecundidad. 1a Ed. México DF: Universidad Nacional Autónoma de México; 1992. 
16. Coloma C. Situación de salud de los pueblos indígenas de Latinoamérica. In: Organización Panamericana de la Salud, Sociedad Canadiense para la Salud Internacional, editores. Los pueblos indígenas y la salud: documento base. Winnipeg: Organización Panamericana de la Salud/Organización Mundial de la Salud; 1993. p. 1-101.

17. Berquó E. Refletindo sobre as questões populacionais neste final de século. Novos Estudos CEBRAP 1999; 55:71-81.

18. Cruz I. Entre a vulnerabilidade e a exclusão: as pessoas portadoras de deficiência física face a nova questão social [Tese de Doutorado]. Rio de Janeiro: Instituto de Medicina Social, Universidade do Estado do Rio de Janeiro; 2001.

19. Fernández M, Bolívar E, Arias MM, Alcaraz G. Estudio demogenético en el suroeste de Antioquia (Municipio de Jardín). Proyecto de Investigación. Medellín: Universidad de Oviedo/Universidad de Antioquia; 1993.

20. Arango R, Sánchez E. Los pueblos indígenas de Colombia. Bogotá: Tercer Mundo; 1998.

21. Arias MM, Gálvez A, Alcaraz G. Situación de salud maternoinfantil en asentamientos emberá. Dabeiba-Antioquia-Colombia 1985-1986. Invest Educ Enferm 1988; VI:23-33.

22. Gobernación de Antioquia. Anuario estadístico de Antioquia. Medellín: Dirección Sistema de Información Geoestadística, Departamento Administrativo de Planeación; 2000.

23. Tonnies F. Community \& Society (Gemeinschaft and Gessellschaft). New York: Harper; 1957.
24. Alcaraz G, Arias MM, Gálvez A. Situación de salud materno-infantil en asentamientos emberá de Dabeiba, Antioquia 1985-1986. Medellín: Universidad de Antioquia/Instituto Colombiano para el Desarollo de la Ciencia y la Tecnología; 1988.

25. Departamento Administrativo Nacional de Estadística. Las estadísticas sociales en Colombia. Bogotá: Departamento Administrativo Nacional de Estadística; 1993.

26. Preston S. The changing relation between mortality and level economic development. Popul Stud (Camb) 1975; 29:157-248.

27. Rico J. Demografía social y salud pública. Cali: Universidad del Valle; 1993.

28. Bongaarts J. A framework for analysing the proximate determinants of fertility. Popul Dev Rev 1978; 4:105-32.

29. Gutiérrez D. Salud reproductiva: concepto e importancia. Washington DC: Organización Panamericana de la Salud; 1996.

30. Arias MM. Comportamiento reproductivo en las etnias de Antioquia - Colombia. Salud Pública Méx 2001; 43:268-78.

31. Módena ME. Madres, médicos y curanderos: diferencia cultural e identidad ideológica. México DF: Casa Chata; 1990

32. Tapia M. La educación de la mujer en la cultura familiar y en la salud de los hijos: reflexiones teóricas y metodológicas. Cuernavaca: Centro Regional de Investigaciones Multidisciplinarias; 1990.

Recibido el $07 /$ Jun/2004

Versión final presentada el 01/Dic/2004

Aprobado el 10/Ene/2005 